Supporting Information

\title{
Imaging and Targeted Antibacterial Therapy using \\ Chimeric Antimicrobial Peptide Micelles
}

Seong-Cheol Park ${ }^{l}$, Changgon $\mathrm{Ko}^{2}$, Hyejin Hyeon ${ }^{2}$, Mi-Kyeong Jang ${ }^{l, *}$, Dongwon Lee ${ }^{2,3, *}$

${ }^{1}$ Department of Polymer Science and Engineering, Sunchon National University,

255 Jungang-ro, Suncheon, Chonnam, 57922, Republic of Korea

${ }^{2}$ Department of Polymer-Nano Science and Technology and ${ }^{3}$ Department of BIN Convergence

Technology, Jeonbuk National University, Baekjedaero 567, Jeonju, Chonbuk 54896, Republic

of Korea

\section{Corresponding Authors}

Dongwon Lee, dlee@chonbuk.ac.kr; Mi-Kyeong Jang, jmk8856@sunchon.ac.kr 


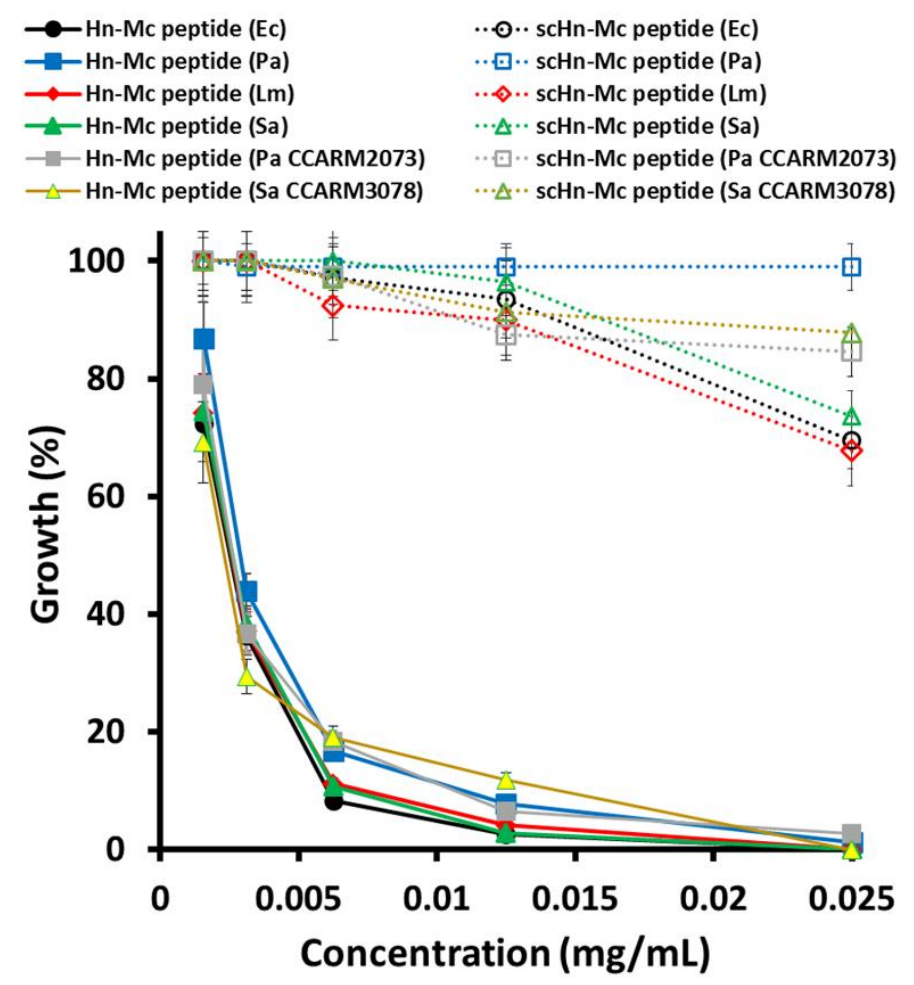

Figure S1. Antimicrobial activity of $\mathrm{HnMc}$ and scramble HnMc peptide in planktonic growth of E. coli $(\mathrm{Ec})$, P. aeruginosa $(\mathrm{Pa})$, L. monocytogenes $(\mathrm{Lm})$, S. aureus $(\mathrm{Sa})$, P. aeruginosa CCARM 2073, and S. aureus CCARM 3078. 

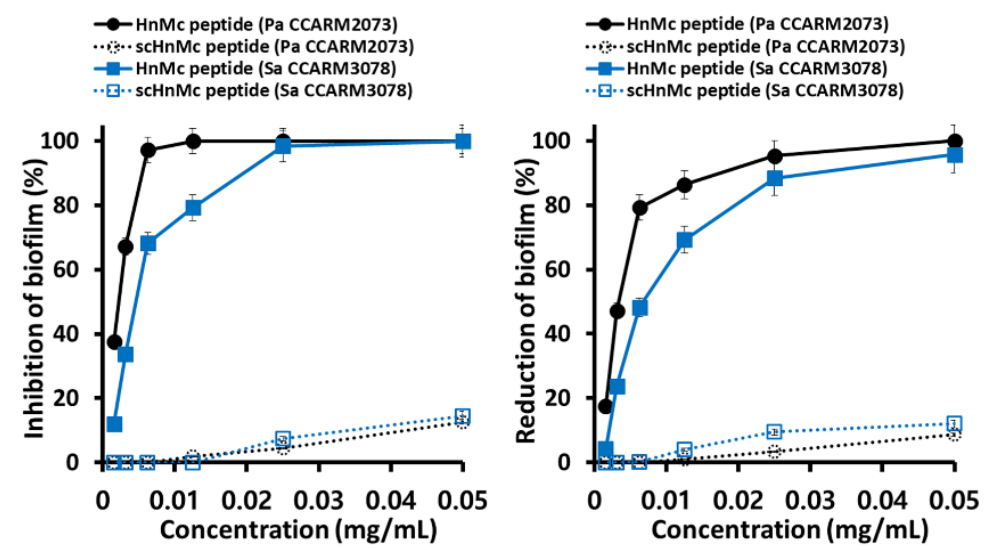

Figure S2. Anti-biofilm activity of $\mathrm{HnMc}$ and scramble $\mathrm{HnMc}$ peptide in biofilm formation and preformed biofilm of drug-resistant $P$. aeruginosa CCARM 2073, and S. aureus CCARM 3078. 


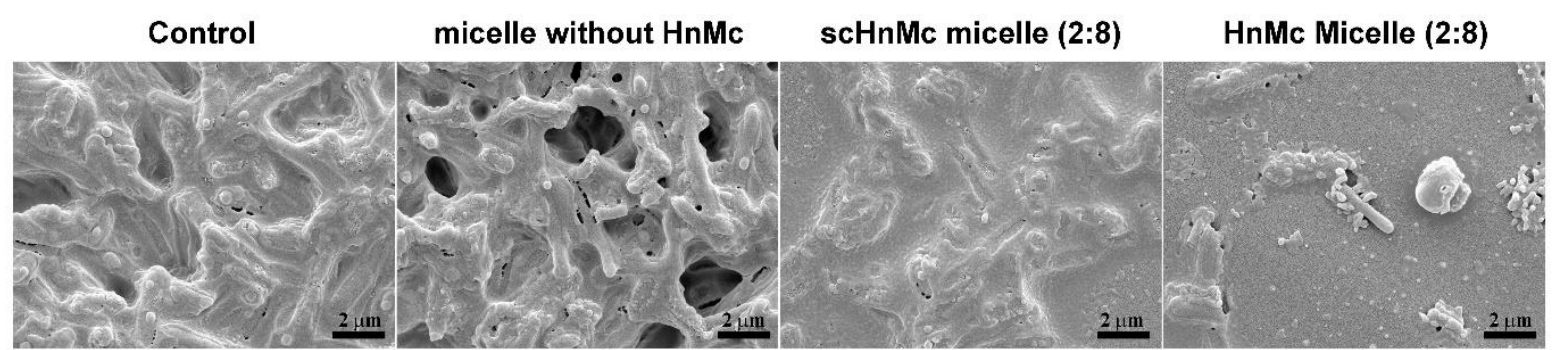

Figure S3. Reductive effects of HnMc micelles in biofilm pre-formed by P. aeruginosa CCARM 2073 on plastic disks. After biofilm formation, micelles were applied at $0.25 \mathrm{mg} / \mathrm{mL}$ for $24 \mathrm{~h}$ and biofilm was observed under SEM. 

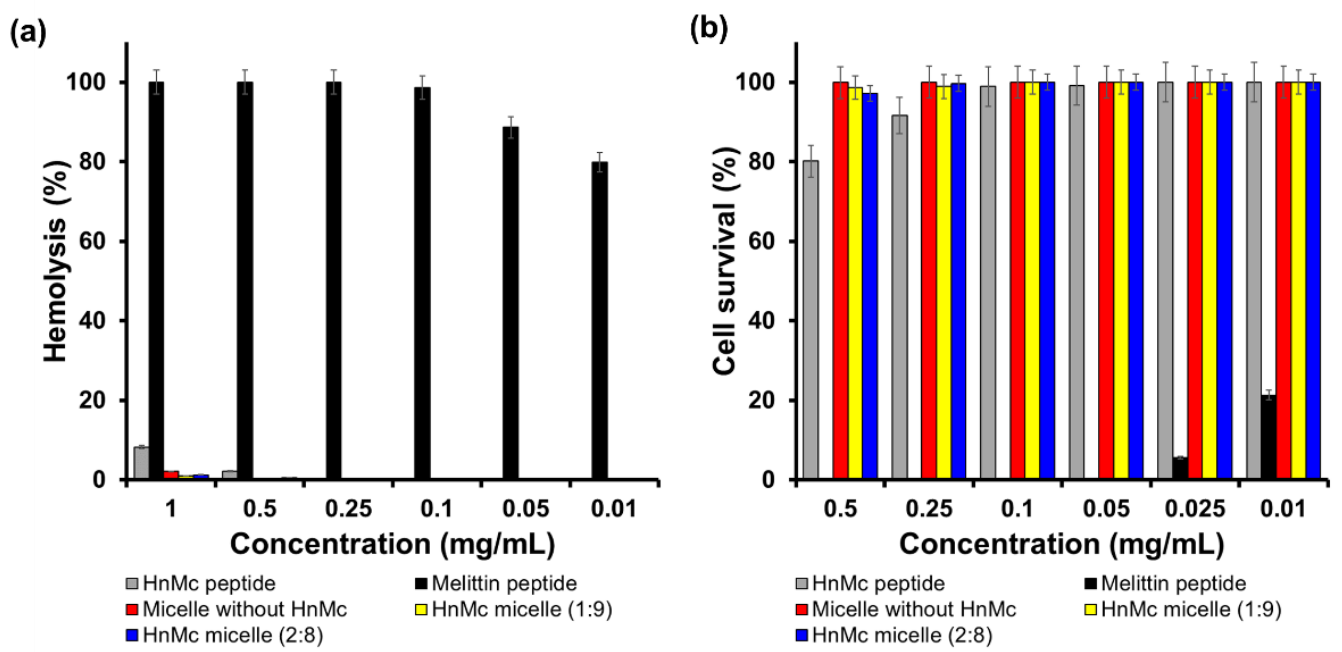

Figure S4. Toxicity of HnMc micelles. (a) Hemolysis of red blood cells. (b) Cell viability of HaCaT cells. Values are mean \pm s.d. $(n=3)$. 

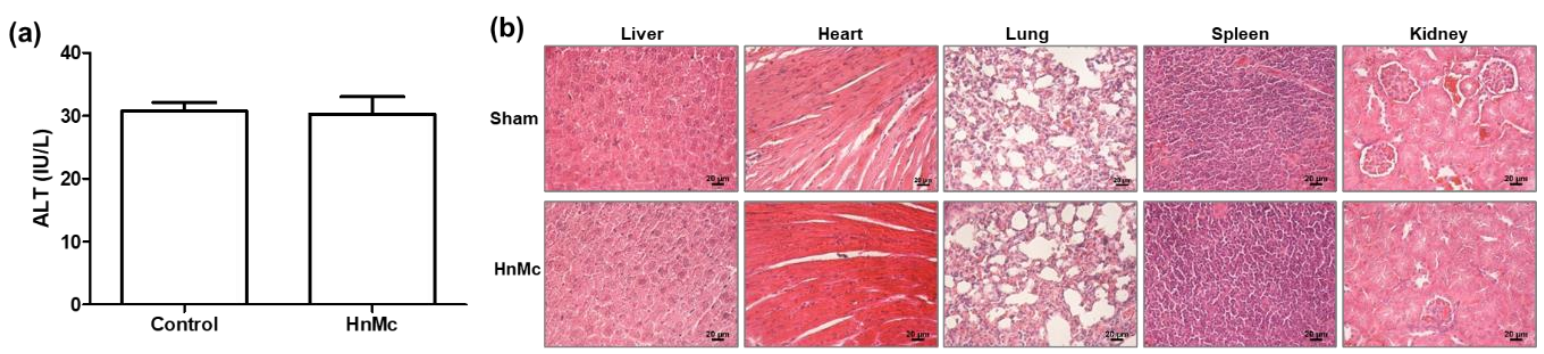

Figure S5. In vivo toxicity of HnMc micelles. (a) The serum level of ALT. Values are mean \pm s.d. $(n=3)$. (b) Histological examination of major organs. 